\title{
Morphometric Deformations of Small Airways and Alveoli under Quasi-static Inflation Process
}

\author{
Toshihiro Sera, Kentaro Uesugi and Naoto Yagi \\ Japan Synchrotron Radiation Research Institute (JASRI/SPring-8)
}

\begin{abstract}
Localized morphometric deformations of small airways and alveoli during respiration have many biomedical and physiological implications. We developed fast synchrotron radiation CT system to visualize the small airways and alveoli of an intact mouse lung without fixation and dehydration, and analyzed their localized morphometric deformations between functional residual capacity (FRC) and total lung capacity (TLC). In the diameter behavior, the averaged and range values were significantly larger for smaller airways (68.8\%, range: $0.36-0.89$ ) than larger airways (45.2\%, range: $0.40-0.57$ ). These results indicated that the airway did not deformed in same manner and that these morphological differences characterized the heterogeneous lung function. $J$ Physiol Anthropol Appl Human Sci 24(4): 465-468, 2005 http://www.jstage.jst.go.jp/browse/jpa
\end{abstract}

[DOI: 10.2114/jpa.24.465]

Keywords: three-dimensional visualization, lung compliance, synchrotron radiation CT, asthma, heterogeneity

\section{Introduction}

In many respiratory diseases, significant compliance abnormalities mainly occur in localized regions of bronchi and bronchioles, and thus the localized deformations based on small scale observations must be identified. Especially, asthma is characterized by chronic inflammation (airway remodeling) of small airway walls (Tashkin, 2002). In the previous works, three methods were reported to evaluate the localized airway deformations. One approach is to visualize dehydrated and fixed preparations by using optical microscopy (Klingele and Staub, 1971; Okazawa et al., 2000). Another approach is to insufflate a metal powder into a lung as a contrast agent and visualized using bronchogram (Hughes et al., 1972; Menkes et al., 1972; Sittipong et al., 1974). The other approach is to measure the mechanical properties of excised small airway segments (Tiddeens et al., 1999) and lung (Sera et al., 2004) in vitro. However, in situ, the intrapulmonay airways are surrounded by lung parenchyma and thorax, and the effective microscopic airway deformation is influenced by the surrounding support (Fig. 1). Recently, we developed the "fast" synchrotron radiation CT system to visualize the small airways and alveoli of intact mouse lung without fixation and dehydration (Sera et al., 2005). In this study, we analyzed the localized airway deformations of mouse lung under quasistatic inflation process and compared the localized airway deformations in healthy control and asthma model mice.

\section{Methods}

Imaging

All protocols of experiment were approved by the SPring- 8 Experimental Animals Care and Use Committee. In this study, control and asthma groups $(n=10)$ were imaged using fast synchrotron radiation $\mathrm{CT}$. Asthma model mice were induced by the previous protocol (Shinagawa et al., 2003). Euthanized mice were mounted on the rotation stage (Fig. 2).

The synchrotron radiation CT system has been constructed in BL38B1 and BL20B2 at SPring-8. SPring-8 is the third generation synchrotron radiation source in Hyogo, Japan.

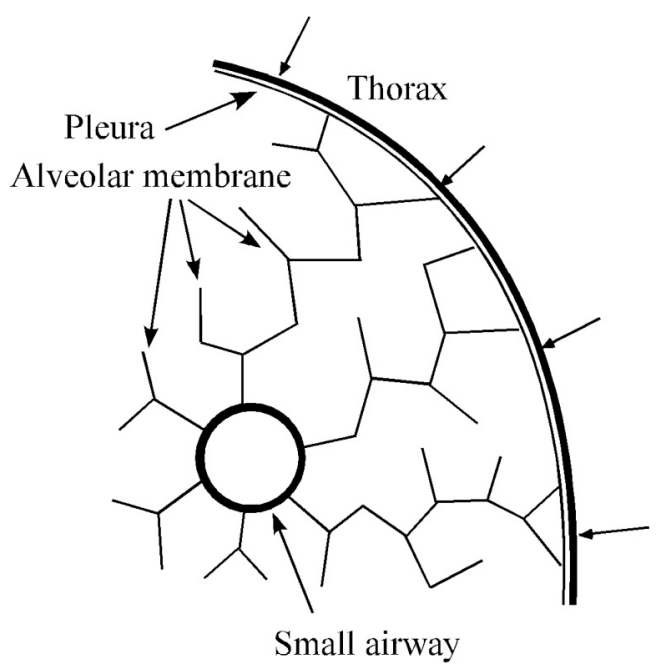

Fig. 1 The schematic model of small airways and alveoli in thorax (Sera et al., 2005). 


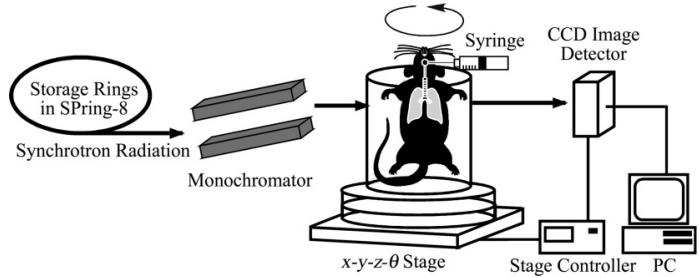

Fig. 2 The experimental apparatus (Sera et al., 2005).

Especially, the experimental hutch 3 in BL20B2 is located 206 meters from the source point and so provides the large field imaging (Goto et al., 2001). The CT system consists of an Xray light source, a double crystal monochromator, a rotation stage, a high-resolution image detector (AA60 and C4880-41S, Hamamatsu Photonics K.K.) and PC (Fig. 2). The X-ray energy and exposure time were set to $20 \mathrm{keV}$ and $250 \mathrm{~ms} /$ projection. The scintillator was $10 \mu \mathrm{m}$-thick at BL38B1 and 20 $\mu \mathrm{m}$-thick at BL20B2 gadolinium oxysulfide doped with terbium $\left(\mathrm{Gd}_{2} \mathrm{O}_{2} \mathrm{~S}: \mathrm{Tb}, \mathrm{P} 43\right)$. The scan time was shorter than $7 \mathrm{~min}$. The size of each pixel was $11.8 \mu \mathrm{m} \times 11.8 \mu \mathrm{m}$.

To analyze the morphometric deformation of the same airway networks, the same branching networks were visualized under quasi-static inflation process. FRC (functional residual capacity) was defined as the lung volume at euthanasia, and the lung volume could be externally regulated using a syringe connected to the trachea. The volume of TV (at end of tidal inspiration) and TLC (total lung capacity) was defined as the sum of FRC +250 and $800 \mu \mathrm{L}$, respectively (Schulz et al., 2002).

\section{Measurement of airway structure}

We first identified the cross-sections of the parent airway and the small bronchus merging into it in 2D CT images. The segmentation was slice-by-slice process that was applied to all slices. And then, to determine the morphometry (diameter $D$ and length $L$ ) of the small airways, we analyzed their crosssections using the 3D thinning algorithm (Toriwaki et al., 2001). This algorithm detects the skeletonized lines and branching points of the airway network without changing the topology of the airways. The diameter and length of the alveolar duct at FRC and TLC were defined as the averaged value of five minor and major axes, because it was difficult to identify the 3D networks of the alveolar duct. The fractions of increase in $L$ and $D\left(\delta_{L}\right.$ and $\delta_{D}$, respectively) were normalized by $L$ and $D$ at FRC. And the airway volume $(V)$ was calculated by the formula $\left(D^{2} \times L \times \pi / 4\right)$, and the volume behavior $\left(\delta_{V}\right)$ was defined as the percentage of $V$ at FRC for TLC.

In this study, the airway segments of all animals were classified into 4 groups using a diameter-based technique (Sera et al., 2005): diameter range at FRC $<200 \mu \mathrm{m}, 200-300 \mu \mathrm{m}$, $300-400 \mu \mathrm{m}$ and $>400 \mu \mathrm{m}$. The morphometric changes were presented as average values $\pm \mathrm{SE}$ as a function of the diameter group ( $\mathrm{n}=28-43$ per diameter range). The values of $p<0.05$ were considered to be statistically significant by Steel-Dwass

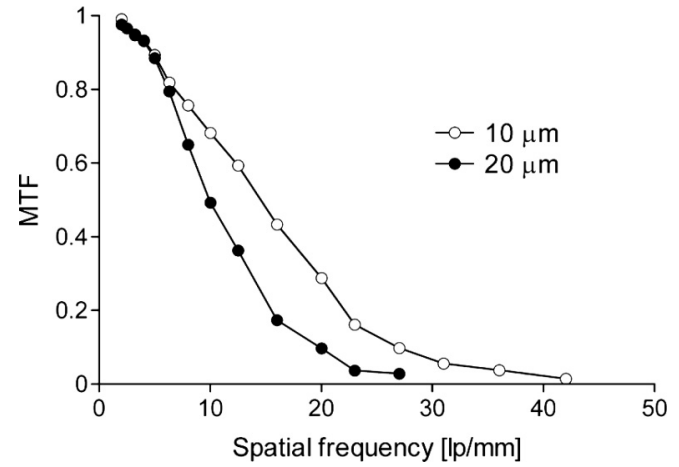

Fig. 3 Modulation transfer function (MTF) of the detector with $10-\mu \mathrm{m}$ and $20-\mu \mathrm{m}$ thick P43 scintillator (Sera et al., 2005). 1 pixel: $11.8 \mu \mathrm{m}$.

comparison.

\section{Results}

\section{Spatial resolution}

For evaluation of the effective spatial resolution, a gold line chart was placed on the front face of the detector with $10 \mu \mathrm{m}$ and $20 \mu \mathrm{m}$ thick scintillator (1pixel: $11.8 \mu \mathrm{m}$ ). Fig. 3 shows modulation transfer function (MTF) for $10 \mu \mathrm{m}$ and $20 \mu \mathrm{m}$ thick scintillator. Since the pixel size is $11.8 \mu \mathrm{m}$, the ultimate resolution is $42.4 \mathrm{lp} / \mathrm{mm}$ (Nyquist frequency). A $20 \mu \mathrm{m}$ thick scintillator blurs the edges, and the spatial resolution was lower.

\section{Localized morphometric deformation of healthy mice}

Using the fast synchrotron radiation CT, not only small airways but also alveoli were visualized without fixation and dehydration (Fig. 4).

The diameter $\left(\delta_{D}\right)$, length $\left(\delta_{L}\right)$, and volume $\left(\delta_{V}\right)$ behavior were presented in each airway group (Fig. 5). $\delta_{D}$ was larger than $\delta_{L}$ for all groups. Further $\delta_{D}$ for smaller airway group was significantly larger than that for larger airways group, although $\delta_{L}$ was not. $\delta_{V}$ was also significantly smaller for smaller airways. Moreover, $\delta_{V}$ for smaller airways group was smaller than the percentage of the lung volume at FRC for that at TLC; $\delta_{V}$ was $28.3 \%$ and the lung volume at FRC was $37 \%$ of that at TLC (Schulz et al., 2002).

\section{Comparison between healthy and asthma model mice}

Figure 6 shows the airway deformations of control and asthma model mouse at FRC and VT. At FRC, the diameter was larger in asthma group than in control group. And the airway deformations were lower in asthma group than in control group.

\section{Discussion}

Using fast synchrotron radiation CT, we first visualized not only small airways but also alveoli of intact lung, and evaluated the localized morphometric deformations based on 
A

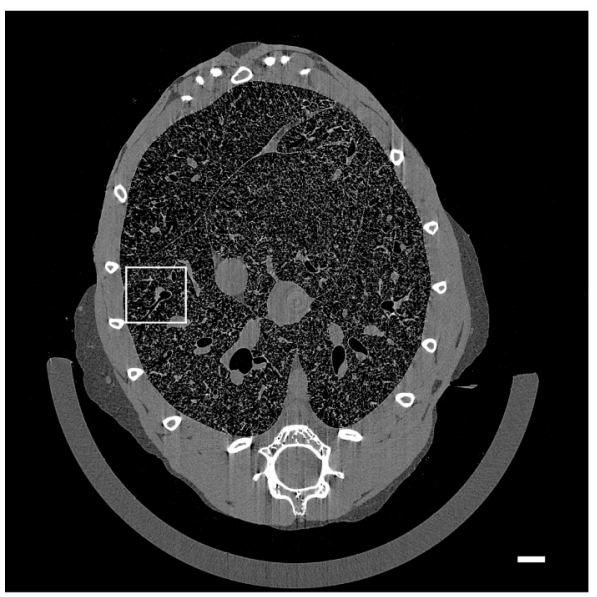

$\mathrm{B}$

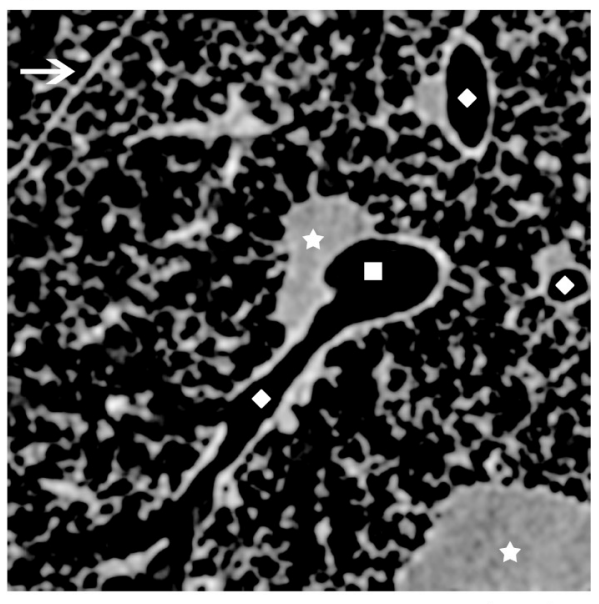

Fig. 4 Representative CT images at BL38B1 (Sera et al., 2005). (A: original images. Bar: $1 \mathrm{~mm}$. B: zoomed images of the square area in A. Square: conducting airways; Star: pulmonary artery; Diamond: small airway; Arrow: interlobaris fissura. Bar: $250 \mu \mathrm{m}$.)

microscopic regions of airway segments under quasi-static inflation process. The high flux synchrotron radiation allows distinguishing the air space from the lung tissue without fixation, dehydration, and contrast agents.

In this study, the airway deformations were analyzed under quasi-static inflation, and all airways did not deform homogenously. $\delta_{V}$ for smaller airways was much smaller than the percentage of the lung volume at FRC to TLC. These results indicated that the volume of the airway segment changed to a greater extent in smaller airways, and that all airways were deformed heterogeneously between FRC and TLC. And for smaller airways, the range of $\delta_{D}$ was larger: $0.40-0.57$ at diameter at $\mathrm{FRC}=400 \pm 10 \mu \mathrm{m}$, and $0.36-0.89$ at diameter at $\mathrm{FRC}=200 \pm 10 \mu \mathrm{m}$. Namely, the small airways of same size did not behave in same manner. These morphological differences between large and small airways and between same size airways characterized the heterogeneous lung function. For example, for small airways and alveoli, traditionally, the airflow is approximately zero and

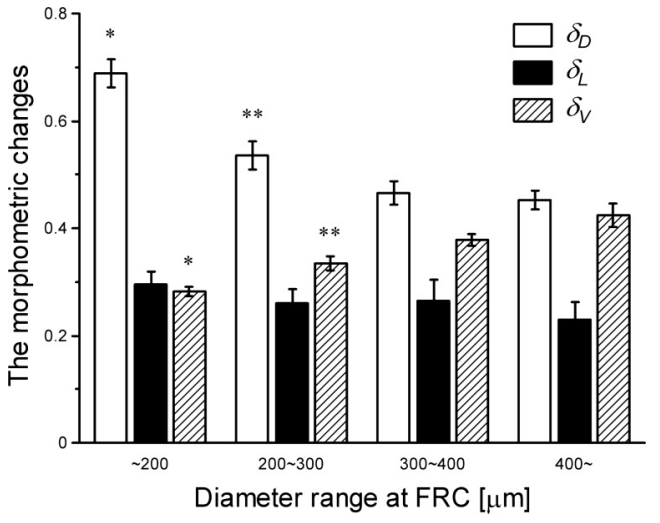

Fig. 5 The morphometric deformations for each airway group. $* \mathrm{p}<0.001$ and $* * \mathrm{p}<0.05$
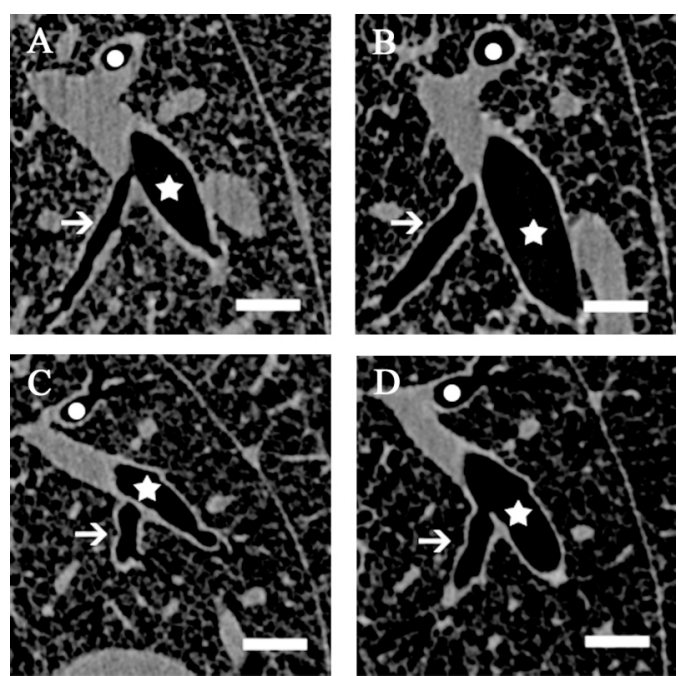

Fig. 6 The zoomed CT images of asthma model (A\&B) and control mice $(\mathrm{C} \& \mathrm{D})$ at FRC $(\mathrm{A} \& \mathrm{C})$ and VT $(\mathrm{B} \& \mathrm{D})$. The diameter increased $24-46 \%$ in asthma mice, and $35-52 \%$ in control mice. Bars: $500 \mu \mathrm{m}$.

gas transport is dominated by simple diffusion (Pedly et al., 1992). However, this dramatic deformation may promote complex diffusion in deep lung. Tsuda et al. (2002) visualized rat lungs in rhythmical ventilation and demonstrated the chaotic mixing, and proposed that this mixing promoted the particle deposition in alveoli (Haber et al., 2003). Ertbruggen et al. (2004) reported that the particle deposition was highly heterogeneous for branches of the same generation in 3D branching networks. We believe that our results supported the previous studies.

The localized mechanical properties of small airways and alveoli are highly affected by the surrounding lung parenchyma and thorax (Fig. 1). The small airways in lung parenchyma were stiffer than the isolated airways, because the lung parenchyma acts as a spring (Sera et al., 2004). Furthermore, compared with the localized deformation of small airways of the excised rat lungs (Sera et al., 2004) ( $D$ was $89 \%$ higher and $L$ was $43 \%$ at TLC), those of intact lung 
was a much smaller deformation ( $D$ was $68.8 \%$ higher and $L$ was $29.5 \%$ higher at TLC) for smaller airways ( $D<200 \mu \mathrm{m}$ at FRC). The intrapulmonary airways, in situ, are surrounded by lung parenchyma and thorax, and the effective microscopic airway deformations are influenced by the surrounding support. The alveolar wall and the thorax act as a spring and wall, respectively.

Compared with control group, the diameter at FRC was larger in asthma group. At FRC the lung volume was larger in asthma group than control group, because the wall distensibility decreased and airway dead space increased. The diameter behavior was lower in asthma group than in control group (Fig. 6), and the difference between the asthma and control group was higher for small airways. This result was consistent with the previous report (Brackl et al., 2000), although their measurements were limited to central airways. We measured the airway compliance of small airways (diameter: $150 \mu \mathrm{m}$ ), and our results suggested that the chronic inflammation occurred in peripheral airways.

In conclusion, the fast synchrotron radiation CT system allowed successful visualization of small airways and alveoli of the intact lung; the airways were embedded in the parenchyma and thorax without dehydration, fixation and contrast agents. Our study is the first to evaluate the localized morphometric deformation using this system, which may have physiological and clinical implications. All airways did not deform in same manner: the airway deformation was larger in smaller airways. These results indicated that airway deformed heterogeneously.

Acknowledgements The experiments were carried out at the SPring-8 with the approval of R\&D Beamline Program Committee and Program Review of SPring-8 (Proposal Number: R03B38B1-0032N, R03B38B1-049N, R04B38N1052N, 2004A0169-NL3-np, 2004B0387-NL3-np).

\section{References}

Brackel HJ, Pedersen OF, Mulder PG, Overbeek SE, Kerrebijn KF, Bogaard JM (2000) Central airways behave more stiffly during forced expiration in patients with asthma. Am J Respir Crit Care Med 162 (3 Pt 1): 896-904

Goto S, Takeshita K, Suzuki Y, Ohashi H, Asano Y, Kimura H, Matsushita T, Yagi N, Isshiki M, Yamazaki H, Yoneda Y, Umetani K, Ishikawa $\mathrm{T}$ (2001) Construction and commissioning of a $215-\mathrm{m}$-long beamline at SPring- 8 . Nucl Instr and Meth A: 467-468, 682-685

Haber S, Yitzhak D, Tsuda A (2003) Gravitational deposition in a rhythmically expanding and contracting alveolus. J Appl Physiol 95: 657-671

Hughes JM, Hoppin FG Jr, Mead J (1972) Effect of lung inflation on bronchial length and diameter in excised lungs. J Appl Physiol 32: 25-35

Klingele TG, Staub NC (1971) Terminal bronchiole diameter changes with volume in isolated, air-filled lobes of cat lung. J Appl Physiol 30: 224-227

Menkes H, Gamsu G, Schroter R, Macklem PT (1972) Interdependence of lung units in isolated dog lungs. J Appl Physiol 32: 675-860

Okazawa M, Paré PD, Lambert RK (2000) Compliance of peripheral airways deduced from morphometry. J Appl Physiol 89: 2373-2381

Pedly TJ, Kamm RD (1991) Dynamics of gas flow and pressure-flow relationship. In Crystal RG, West JB, Barnes PJ, Weibel ER eds. The lung: Scientific Foundations. Paven Press, Philadelphia, 995-1010

Schulz H, Johner C, Eder G, Ziesenis A, Reitmeiner P, Heyder J, Balling R (2002) Respiratory mechanics in mice: strain and sex specific differences Acta Physiol Scand 174: 367-375

Sera T, Uesugi K, Yagi N (2005) Localized morphometric deformations of small airways and alveoli under quasi-static inflation. Respir Physiol Neurobiol (in press)

Sera T, Fujioka H, Yokota H, Makinouchi A, Himeno R, Schtoter R, Tanishita K (2004) Localized compliance of small airways in excised rat lungs using microfocal $\mathrm{x}$-ray tomography. J Appl Physiol 96: 1665-1673

Shinagawa K, Kojima M (2003) Mouse model of airway remodeling: strain differences. Am J Respir Care Med 168: 959-967

Sittipong R, Hyatt RE (1974) Static mechanical behavior of bronchi in excised dog lung. J Appl Physiol 37: 201-206

Tashkin DP (2002) The role of small airway inflammation in asthma. Allergy Asthma Proc 23: 233-242

Tiddens HA, Hofhuis W, Bogaard JM, Hop WC, de Bruin H, Willems LN, de Jongste JC (1999) Compliance, hysteresis, and collapsibility of human small airways. Am J Respir Crit Care Med 160: 1110-1118

Tsuda A, Rogers RA, Hydon PE (2002) Chaotic mixing deep in the lung. Proc Natl Acad Sci USA 99: 10173-110178

van Ertbruggen C, Hirsch C, Paiva M (2005) Anatomically based three-dimensional model of airways to simulate flow and particle transport using Computational Fluid Dynamics. J Appl Physiol 98: 970-980

Received: January 31, 2005

Accepted: March 7, 2005

Correspondence to: Toshihiro Sera, SPring-8/JASRI, 1-1-1

Kouto, Mikazuki, Sayo, Hyogo 679-5198, Japan

Phone: +81-791-58-0833

Fax: +81-791-58-2512

e-mail: sera@spring8.or.jp 